\title{
Das nordische Modell: Antrieb oder Bremse der politischen Entwicklung?
}

Urban Lundberg

Kürzlich erst als Paradebeispiel europäischer Sklerose begraben, kehrt das nordische Modell als exklusive Marke auf den internationalen politischen Markt zurück. Politiker aus der ganzen Welt reisen in die nordischen Länder auf der Suche nach praktikablen Lösungen in Zeiten schnellen wirtschaftlichen und sozialen Wandels. Dennoch bleibt die Frage offen: Ist das nordische Modell eher ein Antrieb oder eine Bremse für die politische Entwicklung? Modelle sind zweischneidige Schwerter im politischen Diskurs. Sie mögen der Politik ein Gefühl für Richtung und Stabilität geben, aber sie können auch zu einem ausgeprägt statischen Blick auf künftige Herausforderungen führen.

\section{Einleitung}

Wenn die Länder Nordeuropas beschrieben werden, endet das gewöhnlich entweder in einer Utopie oder einer Dystopie. ${ }^{1}$ Vielleicht dominieren die positiven Wertungen, aber an einflussreichen Zweiflern und Kritikern hat es nie gefehlt. Die Vorstellung von gut funktionierenden Wohlfahrtsgesellschaften an Europas Peripherie stand immer im Wettbewerb mit der gegenteiligen Behauptung: Dem Bild des Individuums, das dem lähmenden Druck hoher Steuern und einer Kultur ausgesetzt ist, die den Kollektivismus zum allgemeinen Gesetz erhöht hat (Huntford 1971). In der dystopischen Version hat der Norden zur Illustrierung dessen gedient, was der Ökonom Albert O. Hirschman (1991) die drei Thesen der Reaktion genannt hat: dass der Wohlfahrtsstaat vor allen Dingen negative Begleiterscheinungen verursache, dass die durch ihn bewirkten Veränderungen nur kosmetischer Natur seien, oder aber geradewegs ein schlimmeres Übel auslösten Unfreiheit und Wohlstandsverlust (Lindbeck 1997).

Das Hin und Her der Debatte zwischen Utopie und Dystopie kann zurückverfolgt werden bis in die 1930er Jahre, als Schweden sich zum ersten Mal als Pionierland von den anderen nordischen Ländern unterschied. Linke Intellektuelle pilgerten nach Schweden auf der Suche nach Beweisen für die Existenz eines demokratischen Mittelwegs - die Alternative hieß anscheinend nicht zwingend: hier ungehemmter Kapitalismus, dort totalitäre Diktatur (Lundberg/ Tydén 2007). Bekannt wurde das Buch des amerikanischen Journalisten Marcus Childs (1936) über den schwedischen mittleren Weg, dessen Analyse frühzeitig Eingang fand in das Bild der Schweden von sich selbst (Stråth 1993a; Musial 1998). Schritt für Schritt wurden später Norwegen und Dänemark zum Verständnis des Nordens als einer politisch und sozial homogenen Region hinzugefügt, ein Verständnis, das heute auch Finnland und Island umfasst. Die Idee eines besonderen nordischen Modells ist jedoch eine spätere Erfindung. In akademischen Kreisen wurde sie in den 1960er Jahren eingeführt. In der politischen Debatte tauchte sie aber erst in den 1980er Jahren ernsthaft auf (Stråth 1993b).

In den letzten Jahren hat die Diskussion eine etwas andere Richtung genommen. Im Zentrum steht nicht mehr der Wohlfahrtsstaat an sich, sondern es geht um die Krisen- und Reformpolitiken der nordischen Länder seit dem Anfang der 1990er Jahre und wie diese zu bewerten sind. War die Wirtschafts-, Finanz- und Gesellschaftskrise der frühen 1990er Jahre im Norden ein Indiz für die Annäherung des Nordens an das übrige Europa? Oder spiegelte sie gerade die besondere Fähigkeit zur Anpassung und Erneuerung? Bemerkenswert ist hier die Tatsache, dass es zumeist sozialdemokratische Regierungen waren, die den öffentlichen Sektor verkleinerten, Märkte deregulierten und radikale Verwaltungsreformen durchführten (Lundberg 2006). In diesem Zusammenhang entstanden auch neue politische Konzepte und Begriffe wie die dänische „Flexicurity“ in der Arbeitsmarktpolitik und die schwedische Rentenreform von 1994. In beiden Fällen sind die Auswirkungen auf die Organisation gesellschaftlicher Interessen bisher schwer zu überblicken. Eine andere, skeptische Argu- mentationslinie sieht geringe Chancen zur Bewahrung der Tarifautonomie in einem integrierten Europa.

Wie immer man die Reformpolitik der letzten zwei Jahrzehnte bewertet ist letztendlich eine Frage des politischen Standpunkts. Für die Skeptiker bestätigt die historische Entwicklung das Bild einer Arbeiterbewegung, die ihre eigenen Begrenzungen einsehen muss. In dieser Sichtweise hat der Norden bereits durch die oben genannten Reformen sozialdemokratischer Regierungen (Verkleinerung des öffentlichen Sektors, Deregulierung der Märkte etc.) Elemente seiner Eigenart verloren und ist aufgegangen in einer größeren marktliberalen Gemeinschaft. In der positiven Deutung stehen die gleichen Reformen für die Reformfähigkeit des nordischen Modells. Wo viele Industrieländer Schwierigkeiten haben, einen sozialen Konsens herzustellen, der für die Anpassung ihres sozialen Sicherungssystems an neue Bedingungen nötig ist, haben die nordischen

\footnotetext{
Im Gegensatz zur Utopie ist die Dystopie die Beschreibung einer fiktiven Gesellschaft, die sich zum Negativen entwickelt hat.
}

Urban Lundberg, Dr., leitet eine Forschungsgruppe am Institut für Zukunftsforschung in Stockholm. Arbeitsschwerpunkte: Entwicklung des Sozialstaates, Rentenpolitik, Skandinavische Arbeiterbewegung e-mail: urban.lundberg@framtidsstudier.se

Übersetzung aus dem Schwedischen von Adolf Krämer 
Länder aufs Neue bewiesen, dass man die scheinbaren Gegensätze zwischen sozialer Sicherheit und Wachstum respektive Gleichheit und Freiheit überwinden kann. Das nordische Modell mit seiner universalistischen und einkommensabhängigen sozialen Sicherung, seinem durch Tarifautonomie geregelten Arbeitsmarkt und seinen steuerfinanzierten Sozialsystemen wird international zur Referenz. Wenn man Globalisierung mit sozialer Sicherheit und Wettbewerbsfähigkeit in Ländern wie Dänemark und Schweden vereinen kann - wo doch die Ausgangsbedingungen besonders schwierig waren - müsste das auch anderswo gehen.

In diesem Artikel wird versucht, die entstandenen Unklarheiten über die $\mathrm{Zu}$ kunft des nordischen Modells etwas breiter anzugehen. Im Hintergrund stehen die realpolitischen Probleme, die am Horizont aufziehen: alternde Bevölkerungen, Migration, Flexibilität der Arbeitskräfte, Konkurrenz aus Niedriglohnländern. Im Zentrum des Beitrags steht die Frage, welche Rolle der Begriff des nordischen Modells in einem Veränderungsprozess spielen kann, der bereits begonnen hat und vermutlich noch viele Jahre andauern wird. Diese Frage wird auf drei Ebenen diskutiert, die für die Bewahrung des Nordens als besonderem Erfahrungsraum entscheidend sind. Zunächst geht es um die Spielräume der Politik, also Voraussetzungen für ein selbstständiges Verhalten gegenüber den Prozessen, die mit der Globalisierung verknüpft sind (Abschnitt 2 und 3); zum Zweiten um die Geografie des Vergleichs im Sinne der Voraussetzungen zur Aufrechterhaltung des Begriffes „Norden“ als selbstverständlichem Referenzpunkt für die nordischen Länder (Abschnitt 4) und zum Dritten um den Vorrang des Modells, also die Bedeutung der Modellvorstellung für die politische Kreativität, das visionäre Denken und die Fähigkeit zur demokratischen Mobilisierung (Abschnitt 5).

\section{Die Spielräume der Politik}

Die sozialdemokratische Arbeiterbewegung hat immer eine besondere Stellung in der Forschung über die Entwicklung des westlichen Wohlfahrtsstaates gehabt. Man hat lange Zeit ein Gleichheitszeichen gesetzt zwischen der Ausprägung des Wohl- fahrtsstaates und der Stärke und den strategischen Fähigkeiten der Sozialdemokratie. Wo die Sozialdemokraten stark und gut organisiert waren, war der Sozialstaat universalistisch und umfassend, wo sie schwach und zersplittert waren, selektiv oder korporativ. Diese sozialdemokratische Version der Entwicklung hat insbesondere die Forschung über die Entwicklung des Sozialstaates in den nordischen Ländern dominiert. Man kann sagen, dass das nordische Modell ein Beispiel dafür geworden ist, was herauskommt, wenn eine reformistische Arbeiterbewegung die Macht hat, der gesellschaftlichen Entwicklung ihren Stempel aufzudrücken.

Dies spiegelt sich auch in den Schlussfolgerungen des einflussreichen Buches von Gøsta Esping-Andersen (1990) über Wohlfahrtskapitalismen, das mehr als irgendeine andere Untersuchung die nordischen Länder als Fokus in der wissenschaftlichen Diskussion über die Entwicklung des Sozialstaates etabliert hat. Für ihn zeichnet sich das nordische Modell durch eine weitgehende Dekommodifizierung aus, das heißt, die Bürger können im Falle der Unfähigkeit, einer Erwerbstätigkeit nachzugehen (bei Krankheit, Arbeitslosigkeit, Kindergeburt und Invalidität), ihren Lebensstandard beibehalten, ohne von den Kräften des Marktes abhängig zu sein. Hierin unterscheidet sich der Norden vom liberalen Modell der angelsächsischen Länder, die dem privaten Versicherungsgedanken breiten Raum geben und dem korporativen Modell der kontinentaleuropäischen Länder, deren Sozialversicherungssysteme deutlicher an den Arbeitsmarkt und die Berufszugehörigkeit gekoppelt sind.

In der Forschung wird das sozialdemokratische Verständnis gesellschaftlicher Entwicklung in der Formulierung „Politik macht einen Unterschied“ (Korpi 1983) zusammengefasst. Das mag selbstverständlich erscheinen. Tatsache ist aber, dass die Bedeutung der Politik für den Fortgang der Entwicklung immer infrage gestellt worden ist. Korpi hat stets betont, dass in den nordischen Ländern die Einflussmöglichkeiten der Politik auf die gesellschaftliche Entwicklung mehr als in anderen Ländern erhalten blieben und nicht durch vermeintliche Sachzwänge der Industrialisierung bzw. des Kapitalismus zunichte gemacht wurden. Gleichzeitig hat er sich mit dieser Sichtweise gegen eine Überbetonung des Konzeptes gewandt, dass Institutionen den entscheidenden Unterschied machen. Dieses betont den eingeschränkten Handlungsspielraum der Politik aufgrund der „Pfadabhängigkeit“ politischer Entscheidungen innerhalb eines vorgegebenen institutionellen Rahmens.

Was bedeuten also à la longue Macht und Ideen, kurz politische Überzeugungen, wenn sie mit den Wirkkräften des industriellen Wandels oder der Wandlungsfähigkeit des Kapitalismus konfrontiert sind? Andere Forschungsansätze haben eine institutionenzentrierte Interpretation zur Entwicklung der Gesellschaft geliefert. Damit ist gemeint, dass Spielräume der Politik durch Beschlüsse begrenzt werden, die in der Vergangenheit in einem bestimmten institutionellen Rahmen gefällt wurden (Steinmo/Thelen 1992). Aus dieser Perspektive erscheint das nordische Modell nicht selbstverständlich als Resultat politischer Strategie, sondern als ein Sonderweg, den die einzelnen Länder zu Beginn des 20. Jahrhunderts betraten. Wie der schwedische Historiker Klas Amark in einem Buch über die Entstehung des Sozialstaates in Schweden und Norwegen unterstreicht, ist Politik nicht nur „zu wollen“- Olof Palmes berühmtes Wort - „sondern oft genug die Wahl zwischen Möglichkeiten, die man nicht voll unter Kontrolle hat" (Åmark 2005, S. 279).

\section{Ein nordisches Modell in der Europäischen Union}

Politik heißt also beides, „wollen“ und „wählen“. Im Norden hat dies seinen besonderen Ausdruck gefunden. Der politische Wille war in hohem Maße sozialdemokratisch geprägt und die alternativen politischen Wahlentscheidungen traf man auf der Grundlage einzigartiger institutioneller Voraussetzungen. Daraus resultierte eine Gesellschaftsordnung, die sich vom kontinentalen und angelsächsischen Mainstream unterscheidet. Die nordischen Politiker der Zeit zwischen den Weltkriegen kümmerten sich nicht um Modelle. Ihr Einsatz galt dem Gegenteil: von etablierten Schablonen wegzukommen und eine Volksmehrheit zu mobilisieren für zu dieser Zeit originelle Lösungen konkreter Probleme.

Eine Schlüsselkategorie, die in diesem Zusammenhang oft vernachlässigt wird, ist 
der politische Entscheidungsspielraum, den man hat, das heißt die Möglichkeiten zur Willensentwicklung und die Autonomie der Wahlentscheidungen. Was dies betrifft, setzt die Europäische Union (EU) einen neuen Rahmen für das nordische Modell, der die Entscheidungsfreiheit begrenzt. Auf vielen Gebieten - der sozialen Sicherheit, dem Arbeitsrecht und der Besteuerung - gibt es aus nordischer Sicht Bedarf für Minimalnormen, die sicherstellen, dass die Integration nicht im Wettbewerb um die niedrigsten Löhne unterminiert wird. Das Problem bestand bisher darin, dass verbindliche Normen einstimmige Beschlüsse im Ministerrat der EU voraussetzen, was sich historisch als unrealistische Erwartung herausgestellt hat. Selbst die Perspektive, nach der Ratifizierung des Vertrags von Lissabon viele Entscheidungen mit qualifizierten Mehrheiten treffen zu können, ändert da wenig. Diese Lösung kann für die nordischen Länder immer noch zum Bumerang werden, was beispielsweise eine selbstständige Verteilungspolitik angeht oder den Erhalt ihres Verhandlungssystems auf dem Arbeitsmarkt.

Ein anderer Effekt der europäischen Integration ist die Stärkung der Kräfte, die formaljuristische Positionen auf Kosten politischer Entscheidungen in den Vordergrund rücken wollen. Hier ist es die Vielfalt der politischen und administrativen Ebenen innerhalb der EU, die den Bedarf nach einer verbindlicheren Rechtsordnung entstehen lässt. Man geht davon aus, dass nur Gerichte mit weitgehenden Befugnissen die Voraussetzungen erfüllen, um die Koordinierungsprobleme zu lösen, die entstehen, wenn verschiedene politische Systeme im Rahmen eines standardisierten Reglements zusammengeführt werden und zusammenarbeiten sollen (Hirschl 2004). In der zwischenstaatlichen Zusammenarbeit gibt es keinen großen Spielraum für eine öffentliche Debatte, dagegen aber einen großen Bedarf an klaren Normen.

Ein anschauliches Beispiel für die Verrechtlichung auf europäischer Ebene ist der Konflikt im schwedischen Vaxholm 2004, bei dem es um gewerkschaftliche Kampfmaßnahmen des schwedischen Bauarbeiterverbandes gegen ein lettisches Bauunternehmen ging, das für seine Arbeiter keinen schwedischen Manteltarifvertrag unterzeichnen wollte (Zaremba 2006). Diese Angelegenheit, die in den schwedischen Medien breit diskutiert wurde, kam schließlich vor ein schwedisches Arbeitsgericht, das den Fall an den Europäischen Gerichtshof weiterleitete. Das schwedische Gericht wollte insbesondere auf zwei Fragen eine Antwort. Erstens, ob die Kampfmaßnahmen der Gewerkschaft vereinbar waren mit der in der Union garantierten Dienstleistungsfreiheit, zweitens, ob sie vereinbar waren mit dem Verbot der Diskriminierung bei öffentlichen Ausschreibungen (Tamas/ Münz 2006).

Die Entscheidung des Europäischen Gerichtshofs vom 18. Dezember 2007 bedeutete eine Niederlage für die schwedische Gewerkschaftsbewegung und in Teilen eine Infragestellung des Modells von Tarifverhandlungen. Das Gericht erkannte zwar das Recht auf gewerkschaftliche Kampfmaßnahmen an. Das Urteil bedeutet jedoch die Einführung des Prinzips der Verhältnismäßigkeit ins schwedische Arbeitsrecht. Früher war es möglich, die Rechtmäßigkeit von Kampfmaßnahmen von einem schwedischen Gericht überprüfen zu lassen. Nunmehr soll es auch möglich sein, zu prüfen, ob diese angemessen sind (ECJ 2007). Auf die schwedische Wirklichkeit übertragen, besagt das Urteil, dass es Grenzen dafür gibt, wann und wie Streikmaßnahmen durchgeführt werden können und welches Recht Gewerkschaften haben, Forderungen aufzustellen. Und es ist nicht mehr die schwedische Gesetzgebung, die diese Grenzen definiert, sondern es ist EURecht. Die Folge davon ist, dass sich die Balance auf dem schwedischen Arbeitsmarkt zugunsten der Arbeitgeberseite verschieben wird. Eine endgültige Entscheidung des schwedischen Arbeitsgerichts steht noch aus. Es wird jedoch erwartet, dass das Gericht die Entscheidung des Europäischen Gerichtshofes übernimmt. Bereits jetzt ist klar, dass damit zentrale Elemente und Werte, die mit dem nordischen Modell assoziiert werden, in Abhängigkeit von einer europäischen Institution geraten (Europäischer Gerichtshof), auf die die nordischen Länder selbst nur marginalen Einfluss haben.

\section{Die Geografie der Vergleiche}

Wie schon angedeutet, existiert das nordische Modell in erster Linie in der Abgrenzung gegenüber dem Rest der Welt. Wenn man jedoch die nordischen Länder unter- einander vergleicht, ergibt sich auch eine Reihe von Unterschieden. Eine nordische Gruppe von Historikern, die 2006 eine Untersuchung zum nordischen Modell veröffentlichte, kam zu dem Schluss, es handele sich um „ein Modell mit fünf Ausnahmen“ (Christiansen/Markkola 2006, S. 28). Hier ist nicht der Platz, um alle Unterschiede zwischen den nordischen Ländern darzulegen. Sie lassen sich aber an einzelnen Politikfeldern illustrieren. So unterscheiden sich beispielsweise das schwedische und das dänische Rentensystem oder das norwegische und finnische Gesundheitssystem. Erst im Vergleich der nordischen mit anderen Ländern treten die gemeinsamen Züge hinsichtlich der Organisation des Arbeitsmarktes, der Ausformung des Sozialstaates und der Beziehung zwischen den Geschlechtern hervor. Im Vergleich zu anderen Industrienationen weisen die nordischen Länder unter anderem folgende gemeinsamen Merkmale auf: Sie verfügen alle über einen großen und überwiegend steuerfinanzierten öffentlichen Sektor. Die öffentlichen Dienstleistungen und das soziale Sicherheitssystem sind universalistisch und integrativ. Des Weiteren besteht eine weitreichende Autonomie der Tarifparteien, die mit dem Staat und den Institutionen des Arbeitsmarktes im Rahmen der aktiven Arbeitsmarktpolitik eng zusammenarbeiten. Außerdem verfolgen diese Länder eine Familienpolitik, die darauf abzielt, eine größere Geschlechtergleichheit herzustellen.

Jenseits dieser gemeinsamen Merkmale arbeiten die nordischen Länder eng zusammen. Die Formen dieser nordischen Zusammenarbeit und ihre Bedeutung sollen im Folgenden erläutert werden. In der gesellschaftswissenschaftlichen Forschung ist es immer üblicher geworden, sich für die Art und Weise zu interessieren, wie politische Akteure voneinander lernen. Sie treffen sich in internationalen Zusammenhängen und diskutieren über ihre Erfahrungen. Vor größeren Reformen studieren sie ihre jeweiligen Fehler und Erfolge. Zusammenarbeit muss nicht notwendigerweise bedeuten, gemeinsame Lösungen zu entwickeln (das gestaltete sich im Norden eher schwierig). Zusammenarbeit kann auch eine Form für kreative Konkurrenz bedeuten. Verschiedene Länder holen sich ihre jeweiligen Lösungen bei den anderen und passen sie ihren eigenen nationalen Voraussetzungen an. In der Forschung redet man deswegen über das Vergleichen als 
politische Praxis: Politik hat nicht nur mit dem zu tun, was man will und welche Aktivitäten man wählt. Politische Ergebnisse sind auch von der Fähigkeit abhängig, sich in einer Welt von mehr oder weniger nachahmenswerten Beispielen zu orientieren (Kettunen 2006).

Die regionale Zusammenarbeit im Norden ist vermutlich einzigartig in der Welt. Neben den offensichtlichen Formen dieser Zusammenarbeit wie dem Nordischen Rat und dem Nordischen Ministerrat existieren noch andere Formen der $\mathrm{Zu}-$ sammenarbeit auf verschiedenen politischen Ebenen. Die nordischen Länder treten oft in internationalen Organisationen wie den Vereinten Nationen (UN), der Internationalen Arbeitsorganisation (ILO) und der Weltbank und zu einem gewissen Grad auch in der Europäischen Union gemeinsam auf (Kettunen 2006). Im Zusammenhang mit dem nordischen Modell des Sozialstaates muss man in der Nachkriegszeit besonders den Aufbau einer nordischen Sozialstatistik herausstellen, welche die nationalen Akteure dann als Ausgangsmaterial für Vergleiche und Kritik nehmen konnten (Petersen 2006). Besonders in Finnland gewann der Begriff „Norden“ mehr und mehr dynamisches Potenzial. Wie der finnische Historiker Pauli Kettunen gezeigt hat, wurde damit gleichzeitig demonstriert, dass Finnland nicht zum Ostblock gehörte und dass die Zukunft für die finnische Gesellschaft bereits vorgezeichnet war. Zukunft bedeutete in diesem Sinne alles, was in den übrigen nordischen Ländern bereits eingeführt worden war (Kettunen 2001).

Hier nun gibt es Anlass zu näherer Betrachtung. Mit wem vergleicht man sich und was vergleicht man? Wer hat die Macht über die Vergleiche? Heutzutage sind benchmarking und best practice ein Wachstumssektor. Zum Beispiel kann man auf den Lissabonprozess der EU hinweisen, dessen Methode der offenen Koordinierung als Versuch bezeichnet werden kann, einen vergleichenden Kontext zu schaffen. Die EU definiert die Zielvorgaben und gestaltet die Messinstrumente, mit deren Hilfe die Entwicklung in den einzelnen Mitgliedsländern bewertet wird (Telò 2002). Andere Beispiele sind die ständigen Auswertungen der OECD sowie die Arbeit der internationalen Rankingagenturen. Dass die nordischen Länder bei dieser Art von Vergleichen oft gut dastehen, ist kein Widerspruch zu der Tatsache, dass die Kriterien und normativen methodologischen Annahmen der vergleichenden internationalen Studien und Indizes oft im Gegensatz zu den Werten und Eigenschaften stehen, die mit dem nordischen Modell assoziiert werden. Um nur ein konkretes Beispiel zu nennen: Es ist höchst unklar, wie sich die sogenannte Lissabonstrategie, die Europa zur konkurrenzfähigsten Region auf der Welt machen soll, auf zentrale nordische Werte wie Gleichheit, Universalismus, Arbeitsrecht und steuerfinanzierte Wohlfahrtssysteme auswirken wird (Andersson 2004).

Inzwischen kann man sagen, dass sich die nordischen Länder nicht mehr wie früher als relevante geografische Einheit empfinden. Die europäischen und internationalen Zusammenhänge werden immer wichtiger und es fällt immer schwerer, sie $\mathrm{zu}$ ignorieren. Gleichzeitig finden sich die Länder des Nordens heute stärker als früher in wissenschaftlichen Vergleichsstudien, politischen Benchmarking-Prozessen und internationalen Indizes wieder, wobei sie weder auf den jeweils zugrundeliegenden Wertekontext und die methodologische Konzeption der Untersuchungen noch auf deren Ergebnisse einwirken können.

\section{Der Vorrang des Modells}

Die bisherige Argumentation führt letztlich zu Fragen nach dem Modellbegriff selbst. Modell ist ein zweideutiges Wort, das sowohl „Vorbild zum Handeln“ als auch ,vereinfachtes Bild der Wirklichkeit“ bedeuten kann. Bereits auf einem Kongress des Koordinationskomitees der nordischen Arbeiterbewegung in Oslo 1963 schilderte der damalige schwedische Ministerpräsident Tage Erlander den Norden als „ein Ideal für die übrige Welt", was Fragen „der politischen Demokratie, der sozialen Sicherheit und der Freiheit des Einzelnen" angeht. Die Arbeiterbewegung war die treibende Kraft hinter dem, was er als eine ,wirtschaftliche, kulturelle und soziale Revolution" (Lundberg 2006, S. 274) beschrieb. Es war der Blick zurück in die eigene Geschichte und der nach außen in eine von Konflikten geprägte Welt, die es Erlander und anderen nordischen Politikern ermöglichten, ihre eigenen Gesellschaften als Modell zur Nachahmung für andere zu empfehlen, dessen Werte es nach innen zu bewahren und zu verteidigen galt.
Auch dieser Punkt gibt Anlass zum Nachdenken. Als Vorbild für andere mag der Begriff des nordischen Modells visionäres Potenzial haben, aber als vereinfachtes Bild der Wirklichkeit für die nordischen Länder selbst dürfte sich der gegenteilige Effekt einstellen. Die Vorstellung von der Gesellschaft wird statisch, verliert ihre Dynamik. Ein Modell ist stets der Versuch, einen komplexen Zusammenhang auf eine handhabbare Anzahl von Variablen zu reduzieren. Es entsteht eher in Abgrenzung zu anderen Phänomenen als in der politischen Auseinandersetzung. Damit schaffen Modelle sich ihre eigene Problemwelt. Sie werden bedroht, unterminiert und ständig neuen Herausforderungen ausgesetzt. Versuche zur Modifizierung bekommen leicht den Charakter einer Abweichung vom Kern. Ein Modell auf eine sich verändernde Umwelt zu beziehen, gleicht dem Kauf einer Hose für ein Kind im Wachstum. Politik wird so defensiv und auf Reaktion reduziert. Ein Modell landet leicht in einem Zustand permanenter Krise.

Für die Anhänger des nordischen Modells führt dieser Gedanke in eine bestimmte Richtung. Um mit Gøsta EspingAndersen (1985, S. 237) zu sprechen: „Die Zukunft beruht mehr auf dem Sozialstaat, der bereits errichtet worden ist, als auf dem, der versprochen werden kann". Damit gewinnt auch die technokratische Seite (social engineering) der Politik die Oberhand. Ein gutes Beispiel hierfür liefert die schwedische Rentenreform der 1990er Jahre, die von allen involvierten Parteien als die Lösung eines technischen Problems dargestellt wurde, obwohl es sich um eine groß angelegte Reorganisation des gesamten schwedischen Sozialversicherungssystems handelte (Lundberg 2008). Ein derart technokratisches Vorgehen beschränkt nicht nur die Partizipation der Bürger bei weitreichenden, sie betreffenden Reformvorhaben. Es gefährdet auch auf längere Sicht ihre Identifikation mit dem politischen „output" des Systems, mit anderen Worten: mit der Demokratie.

Das nordische Modell bewahren zu wollen, gleicht der Aufgabe, Venedig vor dem Untergang zu retten. Die Aufgabe ist nobel und bewundernswert, jedoch ebenso unsichtbar und undankbar. Im eigentlichen Prozess besteht sogar für den Begriff Reform das Risiko, kompromittiert zu werden. Historisch bezeichnete Reform demokratisch verankerte Veränderungen zum Besseren; immer häufiger werden Reform- 
debatten zu Kontroversen um Kostendruck und Effizienzsteigerung. Um den Preis der Bewahrung eines abstrakten Modells wird der konkrete Alltag der Bürger verändert. Ein Modell ist dann eine Abstraktion und keine Vision. Es blickt nach außen auf die Umwelt und zurück in die eigene Geschichte, nicht nach innen auf die eigenen Bürger und nach vorne, hin zu einer besseren Ordnung.

Nun soll aber die Unterscheidung zwischen Modell und Vision nicht übertrieben werden. Die Werte, die nach allgemeiner Anschauung das nordische Modell ausmachen, behalten ihre Popularität bei den Wählern. Wie die bürgerlichen Parteien in zunehmendem Maße eingesehen haben, ist es so gut wie unmöglich, eine nationale Wahl in den nordischen Ländern zu gewinnen, indem man radikale Alternativen ankündigt. Dies gilt sowohl für die bürgerliche dänische Partei „Venstre“, wie auch für die traditionell konservativen „Moderaten" in Schweden und die Zentrumspartei in Finnland. Diese Parteien sind nicht an der Macht, weil sie etwa eine deutlich konservativere Politik oder radikale Alternativen versprochen haben, sondern weil sie relativ erfolgreich den Sozialdemokraten ihren Alleinvertretungsanspruch für das nordische Modell streitig machen konnten (Lundberg/Petersen 2006).

Wie bereits konstatiert wurde, haben die nordischen Länder sich als erstaunlich wettbewerbsfähig in der globalisierten Wirtschaft erwiesen. Es ist aber nicht mehr der reformistische Weg der Sozialdemokratie zwischen Kapitalismus und Kommunismus, der die Neugier im Rest der Welt weckt. Der heutige utopische (oder dystopische) Norden ist vielmehr ein unpolitisches Wachstumsmodell, das zu gleichen Teilen aus dänischer „Flexicurity“ auf dem Arbeitsmarkt, schwedischem „den Gürtel enger schnallen " beim Haushalt und finnischer Humankapitalproduktion besteht. Hier soll ein weiteres Mal unterstrichen werden, dass es nicht nur die politische Einstellung ist, die sich in den nordischen Ländern verändert hat. Ebenso bedeutsam ist, dass sich in den letzten Jahren der Fokus des allgemeinen Interesses verschoben hat: vom nordischen Modell als machtpolitischem Ausdruck der Stärke der Arbeiterbewegung hin zum nordischen Modell als Ausdruck für eine politische Problemlösungsform für die Herausforderungen der Globalisierung.

Während das nordische Modell in der Zeit zwischen den Weltkriegen und in der
Nachkriegszeit mit sozialdemokratischen Werten assoziiert wurde, ist die gegenwärtige Reformphase ein vermeintlich neutrales Projekt. Das nordische Modell ist zu einem positiv besetzten Markenzeichen mit unklarem Besitzer geworden. Dies beruht nicht allein auf einer bewussten Strategieänderung der bürgerlichen Parteien. Als die sozialdemokratischen Regierungen in den 1990er Jahren ihre Wirtschaftspolitik änderten, an der sozialen Sicherung sparten und den öffentlichen Sektor rationalisierten, wurde es für die Konservativen leichter, diese Politik fortzusetzen (Lundberg/Petersen 2006). Allerdings liegt darin auch die Gefahr, dass die visionären Elemente der Demokratie gleich mit ausrangiert werden. Es geht um die Lust und Bereitschaft zur Teilnahme an den Organisationen, die dazu beigetragen haben, dass der Norden mit progressiven Werten assoziiert wird. In sämtlichen nordischen Ländern haben die politischen Parteien mit sinkenden Mitgliederzahlen und abnehmenden Aktivitäten zu kämpfen (Petersson et al. 2000). Auch wenn das nordische Modell nicht das Ergebnis eines grandiosen Masterplans war, entsprach es doch den Hoffnungen der Bürger, am politischen Diskurs teilzunehmen und Stellung zu beziehen zu den Widersprüchen und Interessenkonflikten in der Gesellschaft.

\section{Fazit}

Das nordische Modell wurde in Abgrenzung zu einer nahen Vergangenheit und einer unruhigen Außenwelt geschaffen. Der Norden war nie eine zur Realität gewordene Utopie, sondern er öffnete einen gewissen Spielraum zwischen Kapitalismus und Kommunismus. Im Laufe der Jahre wurde es immer schwieriger, dieses Bild aufrechtzuerhalten. Der Begriff ist noch immer eine Realität in der politischen und wissenschaftlichen Diskussion, auch wenn er in den letzten Jahren in einem neuen Kontext interpretiert und mit neuem Inhalt gefüllt wurde. Mit jedem weiteren Jahr jedoch weicht die Geschichte weiter zurück und die Welt um uns herum wird immer präsenter. Ein neues Konzept politischer Legitimität entsteht, das weniger Ideen und Interessenkonflikte betont, sondern stärker darauf abhebt, wie Konsens hergestellt und strategische Reformen konzipiert werden können, um einen verschlankten Sozialstaat für das Zeitalter der Globalisierung zu schaffen.

Gleichzeitig taucht das Risiko auf, dass die Arbeit an der „Zukunftssicherung" des nordischen Modells ausschließlich eine Angelegenheit für Experten und professionelle Politiker wird. Dies liegt in der Natur der Aufgabe. Viele Fragen, die auf der politischen Tagesordnung stehen, sind technischer Art und erfordern ein Spezialwissen, das über die Kenntnisse von engagierten Bürgerinnen und Bürgern - die Basis der Demokratie - hinausgeht: alternde Gesellschaften, die Entwicklung des Steueraufkommens, die Koordinierung innerhalb der EU etc. Gegenüber diesen Herausforderungen erscheinen die gängigen demokratischen Prozesse als umständlich und unmodern. Wenn Sachkenntnis einen adäquaten Weg in die Zukunft weist, warum dann einem ungewissen demokratischen Weg folgen?

Ein Ziel dieses Artikels war es zu zeigen, wie der Begriff des nordischen Modells diese Problematik verschärfen kann. Ein Modell erbt man aus der Vergangenheit, es ist selbstverständlich nicht etwas, das man in die Zukunft projizieren kann. Indem man das Verständnis einer Gesellschaft festmacht an einer bestimmten Form und Verfasstheit, entzieht man einer Demokratie möglicherweise ihre visionäre Kraft. Die Zukunft erscheint nicht mehr offen und formbar, sondern absolut und alarmierend. Interessen und Ideenaustausch werden zurückgestellt zugunsten blocküberschreitender Abkommen und technischer Lösungen. Die Politik wird anonymer und die Distanz zwischen Wählern und Gewählten nimmt zu.

Langfristig ist vielleicht der Vorrang des Modells in der nordischen Debatte eine größere Bedrohung für die progressive Identität der Region als die Serie von Veränderungen in unserer Gegenwart, die als Globalisierung bezeichnet wird. Ein Modell an sich ist keine Garantie für politische Kreativität, visionäres Denken oder demokratische Mobilisierung. Im besten Fall ist es ihr Resultat. Im schlimmsten Fall ist es ein Ausdruck für eine Führungsschicht, die, in der Formulierung des britischen Philosophen R. G. Collingwood, fälschlicherweise „die vergänglichen Bedingungen eines bestimmten historischen Zeitalters für die permanenten Bedingungen menschlichen Lebens" hält (Collingwood 1956, S.224). 
Andersson, J. (2004): A Productive Social Citizenship? Reflections on the Concept of Productive Social Policies in the European Tradition, in: Magnusson, L./Stråth, B. (Hrsg.): A European Social Citizenship? Preconditions for Future Policies from a historical Perspective, Bruxelles, S. 69-88 Åmark, K. (2005): Hundra år av välfärdspolitik: Välfärdsstatens framväxt i Sverige och Norge, Umeå

Childs, M. (1936): Sweden the Middle Way, New Haven

Christiansen, N. F./ Markkola, P. (2006): Introduction, in: Christiansen et al. (Hrsg.): The Nordic Model of Welfare: A Historical Reappraisal, Copenhagen, S. 9-29

Collingwood, R. G. (1956): The Idea of History, New York European Court of Justice (ECJ) (2007): The Court of Justice of the European Communities, C-341/05, Laval un Parneri Ltd vs Svenska Byggnadsarbetareförbundet

Esping-Andersen, G. (1985): Politics Against Markets: The Social Democratic Road to Power, Princeton

Esping-Andersen, G. (1990): Three Worlds of Welfare Capitalism, Princeton

Hirschl, R. (2004): Towards Juristocracy: The Origins and Consequences of the New Constitutionalism, Cambridge Hirschman, A. O. (1991): The Rhetoric of Reaction: Perversity, Futility, Jeopardy, Cambridge

Huntford, R. (1971): The New Totalitarians, London

Kettunen, P. (2001): The Nordic Welfare State in Finland, in: Scandinavian Journal of History 26, S. 225-247

Kettunen, P (2006): The Power of International Comparison: A Perspective on the Making and Challenging of the Nordic Welfare State, in: Christiansen et al. (Hrsg.): The Nordic Model of Welfare: A Historical Reappraisal, Copenhagen, S. 31-65

Korpi, W. (1983): The Democratic Class Struggle, London Lindbeck, A. (1997): The Swedish Experiment, Stockholm Lundberg, U. (2006): A Leap in the Dark: From a Large Actor Approach to a Large Area Approach. The Joint Committee of the Nordic Social Democratic Labour Movement and the Crisis of the Nordic Model, in: Christiansen et al. (Hrsg.): The Nordic Model of Welfare: A Historical Reappraisal, Copenhagen, S. 269-297
Lundberg, U./Petersen, K. (2006): Välfärdsstatens seger - politikens död? Spelet om välfärdsstaten i Sverige och Danmark, in: Lundqvist, T. (Hrsg.), Den kreativa staten: Framtidspolitiska tendenser, Stockholm Lundberg, U./Tydén, M. (2007): Stat och individ i svensk välfärdspolitisk historieskrivning, in: Hedin M. et al. (Hrsg.): Staten som vän eller fiende? Individ och samhälle i svenskt 1900-tal, Stockholm

Lundberg, U. (2008): The Democratic Deficit of Pension Reforms: The Case of Sweden, in: Petersen, K./ Petersen, J. H. (Hrsg.): The Politics of Age: Basic Pension Systems in a Comparative and Historical Perspectives, Bruxelles (im Erscheinen)

Musial, K. (1998): Tracing the Roots of the Scandinavian Model: Image of Progress in the Era of Modernization, Florenz $u$. Berlin

Petersen, K. (2006): Constructing Nordic Welfare? Nordic Social Political Cooperation 1919-1955, in: Christiansen et al. (Hrsg.); The Nordic Model of Welfare: A Historical Reappraisal, Copenhagen, S. 67-98.

Petersson, O./Hemes, G./Holmberg, S./Togeby, L./Wängnerud, L. (2000): Demokrati utan partier? Demokratirådets rapport 2000, Stockholm.

Steinmo, S./Thelen, K. (1992): Historical Institutionalism in Comparative Politics, in: Steinmo, S. et al. (Hrsg.): Structuring Politics: Historical Institutionalism in Comparative Analysis, Cambridge UK, S.1-32

Stråth, B. (1993a): Folkhemmet mot Europa: Ett historiskt perspektiv på 1990-talet, Stockholm

Stråth, B. (1993b): Den nordiska modellen: Historisk bakgrund och hur talet om en nordisk modell uppstod, in: Nordisk Tidskrift 69

Tamas, K./Münz, R. (2006): Labour Migrants Unbound?, Stockholm Telò, M. (2002): Governance and Government in the European Union: The Open Method of Coordination, in: Rodrigues, M. J. (Hrsg.): The New Knowledge Economy in Europe: A Strategy for International Competitivness and Social Cohesion, Cheltenham, S. 242-272

Zaremba, M. (2006): Den polske rörmokaren och andra berättelser från Sverige, Stockholm 